\title{
Title: Targeting lipid peroxidation and mitochondrial imbalance in Friedreich's
}

ataxia.

Rosella Abeti ${ }^{1}$, Ebru Uzun ${ }^{1}$, Indhushri Renganathan ${ }^{1}$, Tadashi Honda ${ }^{2}$, Mark A. Pook $^{3}$ and Paola Giunti ${ }^{1}$

${ }^{1}$ Department of Molecular Neuroscience, UCL, Institute of Neurology, Queen Square WC1N 3BG, London, UK

${ }^{2}$ Department of Chemistry, Stony Brook University, Stony Brook, New York 11794, United States

${ }^{3}$ Ataxia Research Group, Division of Biosciences, Department of Life Sciences, College of Health \& Life Sciences, and ${ }^{2}$ Synthetic Biology Theme, Institute of Environment, Health \& Societies, Brunel University London, Uxbridge, UB8 3PH, UK Correspondence must be addressed to: Dr Paola Giunti p.giunti@ucl.ac.uk

\section{Abstract}

Friedreich's ataxia (FRDA) is an autosomal recessive disorder, caused by reduced levels of the protein frataxin. This protein is located in the mitochondria, where it functions in the biogenesis of iron-sulphur clusters (ISCs), which are important for the function of the mitochondrial respiratory chain complexes. Moreover, disruption in iron biogenesis may lead to oxidative stress. Oxidative stress can be the cause and/or the consequence of mitochondrial energy imbalance, leading to cell death. Fibroblasts from two FRDA mouse models, YG8R and KIKO, were used to analyse two different categories of protective compounds: deuterised poly-unsaturated fatty acids (dPUFAs) and Nrf2-inducers. The former have been shown to protect the cell from damage induced by lipid peroxidation and the latter trigger the well-known Nrf2 antioxidant pathway. Our results show that the sensitivity to oxidative stress of YG8R and KIKO mouse fibroblasts, resulting in cell death and lipid peroxidation, can be prevented by d4-PUFA and Nrf2-inducers (SFN and TBE-31). The mitochondrial 
membrane potential $\left(\Delta \Psi_{\mathrm{m}}\right)$ of $\mathrm{YG8R}$ and KIKO fibroblasts revealed a difference in their mitochondrial pathophysiology, which may be due to the different genetic bases of the two models. Suggesting that variable levels of reduced frataxin may act differently on mitochondrial pathophysiology and that these two cell models could be useful in reflecting the observed differences in the FRDA phenotype. This may reflect a different modulatory effect towards cell death that will need to be investigated further.

Keywords: Friedreich's ataxia, lipid peroxidation, mitochondria, oxidative stress; polyunsaturated fatty acids, nuclear factor E2 related factor.

\section{Introduction}

Friedreich's Ataxia (FRDA) is the most common form of hereditary ataxia (Jones et al. 2012; Koeppen \& Mazurkiewicz, 2013; Parkinson el at., 2013). It is an autosomal recessive disorder found mainly in Caucasians (Richardson et al., 2012), with a prevalence in the European population of 1: 50,000 (Velasco-Sánchez, 2011; see review Wilson, 2012). However, this ratio may vary depending on the population studied (Vankan et al., 2013). The disease is characterized by a progressive sensory and cerebellar ataxia, as both proprioceptive and spinocerebellar fibers are lost (Velasco-Sánchez et al., 2011; Parkinson el at., 2013). Cardiomyopathy, and/or diabetes may also occur in some patients (Pandolfo, 2009; Delatycki \& Corben, 2012; Parkinson et al., 2013). FRDA is characterised by the decrease of frataxin expression, which is due to the transcriptional silencing of the FXN gene. Thus, 
causing mitochondrial iron accumulation and increased oxidative stress (see review Wilson, 2012; Schulz and Pandolfo, 2013).

The strategic approaches to treat FRDA have so far included agents which counteract iron accumulation (Kakhlon et al., 2008; see review Martelli et al., 2012), promote frataxin expression (Herman et al., 2006; Velasco-Sánchez et al., 2011; Libri et al., 2014; Perdomini et al., 2014) or diminish hypersensitivity to reactive oxygen species (Perlman, 2012; Shan et al., 2013; Parkinson et al., 2013). Although some compounds have shown promising beneficial effects, no cure has yet been found for this debilitating disease.

The hypersensitivity to oxygen radicals is one of the major causes of cell death. Therefore, we investigated if deuterised poly-unsaturated fatty acids (dPUFAs) and Nrf2-inducers (antioxidants) could act as protective compounds against cell death and lipid peroxidation increase, in YG8R and KIKO FRDA mouse fibroblast cell models (Al-Mahdawi et al., 2004; Al-Mahdawi et al., 2006; Sandi et al., 2014; Miranda et al., 2002). We then investigated the mitochondrial membrane potential $\left(\Delta \Psi_{\mathrm{m}}\right)$ and found that the two models show a different mitochondrial dysfunction, possibly due to their difference in GAA repeat expansion mutations (YG8R have 190 and 90 , while KIKO have 230). YG8R cells showed a depolarization of $\Delta \Psi_{\mathrm{m}}$, which has been found to be a mild impairment in this model (Abeti et al., unpublished data), while KIKO showed an hyperpolarization of $\Delta \Psi_{\mathrm{m}}$, which could results in a more severe defect. The differences in the two models could in part recapitulate FRDA patient phenotypic variability and therefore be helpful for future drug screening.

\section{Materials and Methods:}


Cell Culture. Primary fibroblasts isolated from kidney of WT (C57BL6/J), Y47R, YG8R and KIKO mice were cultured in DMEM supplemented with $10 \%$ fetal bovine serum (FBS) and $1 \%$ penicillin/streptomycin and glutamine and maintained at $37^{\circ} \mathrm{C}$ in a humidified incubator with $5 \% \mathrm{CO}_{2}$.

Immunocytochemistry. Cultured fibroblasts were fixed with $4 \%$ paraformaldehyde (pH 7.4) in PBS, at RT for 15 min and washed three times with PBS. Cells were permeabilized with $0.1 \%$ Triton $\mathrm{X}-100$ for $30 \mathrm{~min}$ and then blocked in $10 \%$ goat serum, $2 \%$ bovine serum albumin (BSA) and $0.01 \%$ Triton $\mathrm{X}-100$ in PBS and then incubated with primaries antibodies: mouse anti-hFrataxin antibody [18A5DB1]; 1:100 in blocking solution (Abcam) for Y47R and YG8R, mouse anti-mFrataxin for WT and KIKO 1:100 in blocking solution (Abcam). Rabbit polyclonal apoptotic inducing factor (AIF) as a mitochondrial marker (1:250; Abcam), Phalloidin $633 \mathrm{~nm}$ (1:300) and $300 \mathrm{nM}$ DAPI. The secondary antibodies used were: anti-mouse Alex Fluor 568 nm (red; molecular probes; 1:500 dilution in blocking solution), anti-rabbit Alexa Fluor 488 nm (green; molecular probes; 1:500 dilution in blocking solution). Fluorescence images were acquired with a Zeiss LSM 710 confocal microscope using an inverted 40× NA 1.2 objective. The fluorescence intensity form frataxin was measured cell by cell and averaged.

Imaging: Cells were plated on glass coverslips $\left(5 \times 10^{5}\right.$ cells in each well) 24 hours prior to the experiments. Afterwards, cells were pre-treated differentially with: $100 \mu \mathrm{M}$ L-1, L-2, d2-PUFA, d4-PUFA, 50 nM SFN or 50 nM TBE-31, for 24 hours and then incubated briefly in $1 \mathrm{mM} \mathrm{H}_{2} \mathrm{O}_{2}$. Coverslips were mounted on microscopy chambers with $250 \mu \mathrm{L}$ clear HBSS. Lipid peroxidation: Lipid peroxidation was estimated by using C11-BODIPY (581/591) (Molecular Probes). Cells were incubated with $10 \mu \mathrm{M}$ C11-BODIPY (581/591) for 10 minutes at RT. C11-BODIPY (581/591) was excited 
using the 488 and $563 \mathrm{~nm}$ laser line, and fluorescence measured with two band pass, 505 to $550 \mathrm{~nm}$ and 570 and $630 \mathrm{~nm}$. Images were recorded every $10 \mathrm{~s}$ using a Zeiss 710 CLSM confocal microscope. The rate of the resulting slope was calculated cell by cell. Mitochondrial membrane potential assays: The basal mitochondrial membrane potential $\left(\Delta \Psi_{\mathrm{m}}\right)$ level was measured with tetramethyl rhodamine methyl ester (TMRM, $25 \mathrm{nM}$, Invitrogen), by exciting the fluorophore at $560 \mathrm{~nm}$ and collecting the images with a $590 \mathrm{~nm}$ long pass filter. With Z-stacks configuration the fluorescence peaks from the mitochondrial network were collected and analysed. The maintenance of $\Delta \Psi_{\mathrm{m}}$ was measured by using TMRM in "redistribution mode" (Duchen et al., 2003). After the equilibration of the dye, which was kept continuously in the solution, $2 \mu \mathrm{g} / \mathrm{mL}$ oligomycin, $1 \mu \mathrm{M}$ rotenone and $1 \mu \mathrm{M}$ Fccp were sequentially added. TMRM distributes between cellular compartments in response to different potentials and, at concentrations $<50 \mathrm{nM}$, in healthy cells the fluorescent signal shows a mitochondrial localisation, where is retained until mitotoxins induced depolarization.

Cell viability: Cells were plated on 96 well plates, each well with 15,000 cells. Compounds were added for 24 hours, and then incubated for 2 hours with $1 \mathrm{mM}$ $\mathrm{H}_{2} \mathrm{O}_{2} .20 \mu \mathrm{L}$ of MTT $(500 \mu \mathrm{g} / \mathrm{ml})$, 3-(4,5-dimethylthiazol-2-yl)-2,5-diphenyltetrazolium bromide, was added to each well and incubated for 3 hours. MTT was then discarded and the obtained formazan crystals were dissolved in $100 \mu \mathrm{L}$ dimethyl sulfoxide (DMSO) to be read at the spectrophotometer at $570 \mathrm{~nm}$.

\section{Results:}

Frataxin expression levels in FRDA fibroblasts 
The levels of frataxin expression were assessed in fibroblasts of KIKO and YG8R FRDA mouse models, compared with their appropriate controls: WT and Y47R. The KIKO mouse contains 230 GAA repeat expansion mutation within the mouse Fxn gene. The YG8R mouse, instead, contains a human FXN genomic transgene with GAA repeat expansion mutations (190 and 90 GAAs), whereas the Y47R mouse has only 9 GAAs, and both mice are homozygous for the mouse frataxin knockout (Pook et al., 2001; Cossee et al., 2000). By using immunofluorescence, we detected a difference in frataxin levels between WT and KIKO, Y47R and YG8R cells. Frataxin expression is in KIKO, compared to WT and in YG8R compared to Y47R (Fig. 1A). A quantitative analysis of the immunofluorescence data obtained by confocal microscopy confirmed that the differences in frataxin level were significant, showing that $\mathrm{KIKO}$ has $62.4 \pm 16.8 \%$ frataxin and that $\mathrm{YG} 8 \mathrm{R}$ has a $39.18 \pm 10.03 \%$ frataxin levels compared respectively to WT and Y47R fibroblasts $\left({ }^{* *} p<0.001 ;{ }^{* * *} p<0.0001\right.$; Fig.1B).

\section{Preventing cell death in FRDA fibroblasts}

Fibroblasts from YG8R and KIKO mice were cultured and the effects of d-PUFAs and Nrf2-inducers were analysed for cell death. The cells were pre-treated with the protective compounds for 24 hours, and subsequently challenged with oxidant $\left(\mathrm{H}_{2} \mathrm{O}_{2}\right.$ $1 \mathrm{mM})$, in order to measure cell death. Four types of PUFAs were used, two containing hydrogen and two containing deuterium: Linoleic acid-1 and Linolenic acid-2 and d2-PUFA and d4-PUFA, respectively (Fig. 2; Angelova et al., 2015). Although they are all classified as polyunsaturated fatty acids, previous studies have showed that those manipulated with deuterium are more 
effective antioxidants than those containing hydrogen (Shchepinov et al., 2011). The isotopic reinforcement by deuteration of the bisallylic sites, which are most susceptible to oxidation in PUFAs, should provide at least partial protection against oxidative stress in particular, at the membrane levels, such as lipid peroxidation. We also tested the protective effect of two compounds, Sulphoraphane (SFN; Fig.2), and Tricyclic bis(cyanoenone) (TBE-31; Fig. 2) (Honda et al., 2011) that trigger the nuclear factor-E2-related factor-2 (Nrf2) antioxidant pathway. Nrf2 is a transcription factor of the antioxidant response elements (ARE). Under physiological conditions, Nrf2 is kept in the cytosol by a cytoskeletal protein known as Keap 1. However, during oxidative stress, Keap 1 releases Nrf2, which then translocates to the nucleus and activates ARE, leading to the expression of antioxidant enzymes (Hayes et al., 2010; Shan et al., 2013). Our cell viability experiments first showed that YG8R and KIKO fibroblasts both exhibit significantly increased sensitivity to $\mathrm{H}_{2} \mathrm{O}_{2}$. We then showed that this effect was prevented by application of d4-PUFA, SFN and TBE-31 to YG8R fibroblasts (Fig. 3A; YG8R $\mathrm{H}_{2} \mathrm{O}_{2} \quad 180 \% \pm 5$, d4-PUFA 99\% \pm 4.2 , SFN $95 \% \pm 4.8$ and TBE31 98\% $\pm 4.3 ;{ }^{* *} p<0.005 ; n=6$ independent experiments for each condition) or by application of d2-PUFA, d4-PUFA, SFN and TBE-31 to KIKO fibroblasts (Fig. 3B; $\mathrm{KIKO} \mathrm{H}_{2} \mathrm{O}_{2} \quad 170 \% \pm 4.1$, d2-PUFA $56 \% \pm 3.4$, d4-PUFA $67 \% \pm 4.6$, SFN $72 \% \pm 4$ and TBE31 80\% $\pm 4.4 ;{ }^{* *} p<0.005 ; n=6$ independent experiments for each condition). In contrast, the hydrogenated PUFAs (L-1 and L-2) did not display any significant protection against cell death. These findings show that d4-PUFA, SFN and TBE-31 have a protective effect on fibroblasts from FRDA models, preventing cell death. We then wondered if the beneficial effect of these compounds on FRDA was induced by preventing lipid peroxidation. 


\section{Lipid peroxidation in FRDA fibroblasts}

To investigate lipid peroxidation as a cause of death, we analysed YG8R and KIKO fibroblasts with C-11 BODIBY (581/591). Cells were loaded and then challenged with the oxidant to induce oxidative stress $\left(1 \mathrm{mM} \mathrm{H}_{2} \mathrm{O}_{2}\right)$.

In both FRDA cell types lipid peroxidation was increased compared to their respective controls (Y47R and WT), both in untreated and in $\mathrm{H}_{2} \mathrm{O}_{2}$ treated cells. Interestingly, d4-PUFA, SFN and TBE-31 were all found to prevent peroxidation in both YG8R and KIKO cells (Fig.4A; YG8R 230\% \pm 2.6 , YG8R $\mathrm{H}_{2} \mathrm{O}_{2} 320 \% \pm 3$, d4-PUFA $150 \% \pm 3.2$, SFN $135 \% \pm 6$ and TBE31 110\% $\pm 4, ;{ }^{*} p<0.05,{ }^{* *} p<0.005 ; n=6$ independent experiments for each condition; Fig.4B; KIKO 184 \pm 1.5 , $\mathrm{KIKO} \mathrm{H}_{2} \mathrm{O}_{2} 301 \% \pm 2.3$, d4PUFA $110 \% \pm 3.2$, SFN $120 \% \pm 2.1$ and TBE31 $105 \% \pm 4 ;{ }^{* *} p<0.005,{ }^{* * *} p<0.005 ; n=6$ independent experiments for each condition).

\section{$\Delta \Psi_{m}$ in FRDA fibroblasts.}

As mitochondria are known to be affected in FRDA, we then investigated mitochondrial function by looking at $\Delta \Psi_{\mathrm{m}}$, which was used as a read out of mitochondrial health. We first measured the basal level of the $\Delta \Psi_{\mathrm{m}}$ in control and FRDA mouse cells. Interestingly, the YG8R and KIKO models seem to reflect a different type of mitochondrial pathophysiology. Analysing the basal level of $\Delta \Psi_{\mathrm{m}}$, YG8R fibroblasts showed depolarization, while KIKO fibroblasts showed an increased $\Delta \Psi_{m}$ (Fig. 5 A-B; YG8R 70\% \pm 4.6 ; ${ }^{*} p<0.05 ; n=6$ independent experiments; KIKO 133.5 $\pm 3.4 ;{ }^{*} p<0.05 ; n=6$ independent experiments). By pre-treating the cells with $d$ - 
PUFA and Nrf2-inducer compounds and we found that the detrimental mitochondrial phenotype in YG8R cells was recovered by d4-PUFA, SFN and TBE-31 (Fig.5 A-B). However, no such protective effect was identified with KIKO cells; indeed, SFN was shown to exacerbate the $\Delta \Psi_{\mathrm{m}}$ hyperpolarisation (Fig.5 B). The different phenotypes and responses to treatment of the two FRDA mouse models may in part be due to the fact that one model has reduced levels of human transgenic frataxin (YG8R), while the other has reduced levels of endogenous mouse frataxin (KIKO) and a larger GAA repeat expansion.

By looking at the dynamic signal of the $\Delta \Psi_{\mathrm{m}}$, using mitotoxins to analyse the ability of the cells to maintain the mitochondrial potential we found, again, that YG8R cells were depolarised, under administration of oligomycin (inhibitor of the ATP synthase) whilst KIKO were slightly hyperpolarised (Fig. 5 C-D). By using d4-PUFA, SFN and TBE-31, only YG8R showed a significant difference with cells pre-treated with the protective drugs (Fig. $5 \mathrm{E}$; 0.81 \pm 0.017 YG8R; 1.09 \pm 0.15 YG8R-d4PUFA; $1.027 \pm 0.13$ YG8R-SFN; $1.04 \pm 0.11$ YG8R-TBE-31; $\left.{ }^{*} p=0.0074\right)$. KIKO cells did not show any significance of the differences in $\Delta \Psi_{\mathrm{m}}$ (Fig. $5 \mathrm{~F} ; 1.18 \pm 0.14 \mathrm{KIKO} ; 1.12 \pm 0.15 \mathrm{KIKO}$ d4PUFA; $1.10 \pm 0.11$; KIKO-SFN; $1.16 \pm 0.12$ KIKO-TBE-31), suggesting that, perhaps both models show mitochondrial sensitivity to the lowering of frataxin expression but that the cell death pathway is triggered in two different ways.

\section{Conclusion}

FRDA is a neurodegenerative disorder caused by an intronic GAA repeat expansion in the FXN gene, which leads to its transcriptional silencing and the decreased expression of the protein frataxin. The function of this protein is still under 
investigation, however, there is evidence that it is involved in cellular iron homeostasis and ISC biogenesis, which has an effect on enzymes of the mitochondrial electron transport chain (complexes I, II and III) (Gonzáles-Cabo et al., 2005) and aconitase (Bulteau et al., 2004). The main biochemical defects that frataxin deficiency leads to are observed in the mitochondria in the form of iron accumulation and high sensitivity to oxidative stress (Barnham et al., 2004). Oxidative stress has an important role in the degeneration of the specific neurons involved in the disease. Based on insights of frataxin function and the major biochemical defects in FRDA, several therapeutic methods have been investigated. There have been attempts to tackle the iron accumulation by using iron chelators (Goncalves et al., 2008, Pandolfo $M$ et al 2014). Increasing frataxin gene transcription by applying HDAC inhibitors (Hebert, 2008; Libri et al., 2014; Soragni et al., 2014) has been found to be promising, whereas treatment with erythropoietin did not show significant frataxin upregulation (Mariotti el al 2013). Part of the clinical research on FRDA is considering the use of antioxidants, which seem to be the less invasive approach compared to other drugs with more side effects. The present work shows that fibroblasts of FRDA mouse models have a hypersensitivity to oxidation, which can be prevented by reducing lipid peroxidation or activating the Nrf2 pathway. The differences in the length of GAA repeats and the different genetic backgrounds of the two models (one is based on human frataxin and the other on mouse frataxin), may be the cause of the observed mild (YG8R) and severe (KIKO) mitochondrial bioenergetics defects. This different behaviour could perhaps reflect a different modulatory effect produced by the inhibitor factor 1 (IF1), a protein that modulates ATP synthase (Campanella et al., 2008). Therefore, further investigation in these models will examine the presence and the modulatory effect of IF1 in order to better 
understand the signalling involved in the two models, which ultimately may lead to a more specific target to treat patients with FRDA.

\section{Acknowledgments}

RA is supported by FARA and GoFar, A\&C Simeone Foundation. PG has received funding from FARA and GoFar, A\&C Simeone Foundation and the European Union Seventh Framework Programme (FP7/2007-2013) under grant agreement number 242193/EFACTS. PG is supported by the National Institute for Health Research University College London Hospitals Biomedical Research Centre. EU and IR were supported by MSc scholarships. We would like to thank R. Molinari and M. Shchepinov (Retrotrope, Inc.) for D-PUFAs compounds, and Dr Albena DinkovaKostova (University of Dundee) and Prof. Tadashi Honda (Stony Brook University, USA) for Nrf2-inducers.

\section{Figures}

Figure 1: Frataxin levels in fibroblasts of FRDA mouse models. A. Mouse frataxin was measured in WT and KIKO fibroblasts; (a, e) blue nuclear staining Dapi; red, monoclonal mouse antibody against mouse (for WT and KIKO) and human (for Y47R and YG8R) frataxin; green, apoptotic inducing factor (AIF) as a mitochondrial marker; light blue, phalloidin staining the cytoskeleton. WT and KIKO are depicted in 
Panel A I and II, while III and IV show Y47R and YG8R fibroblasts. (Scale bar 10 $\mu \mathrm{m})$. B. Quantitative analysis of fluorescence was made cell by cell and reported on the histogram, which confirms that both KIKO and YG8R cells have a decrease of frataxin compared to their controls (respectively WT and Y47R; ${ }^{* *} \mathrm{p}<0.001 ;{ }^{* *} \mathrm{p}<$ 0.0001).

Figure 2: Protective compounds. A. Linoleic and Linolenic acid. B. Poly unsaturated fatty acid with two and four deuterium. C. Sulpuraphane (SFN) and. Tricyclic bis(cyanoenone) (TBE-31).

Figure 3: Preventing oxidative stress-induced cell death in FRDA fibroblasts.

A. The histogram shows the percentage of cell death after treatment with $1 \mathrm{mM} \mathrm{H}_{2} \mathrm{O}_{2}$ and pre-treatment with PUFAs (L-1 and L-2), d-PUFAs (d2-PUFA and d4-PUFA), SFN and TBE-31. Cell death induced by $\mathrm{H}_{2} \mathrm{O}_{2}$ (between untreated and $\mathrm{H}_{2} \mathrm{O}_{2}$; $\left.{ }^{* *} p<0.005\right)$ in YG8R fibroblasts can be significantly prevented by the pre-treatment (24 h) with $100 \mu \mathrm{M}$ d4-PUFA or Nrf2- inducers (50 nM SFN and $50 \mathrm{nM}$ TBE-31; ${ }^{* *} \mathrm{p}<0.005$ ). B. The histogram shows that after treatment with $\mathrm{H}_{2} \mathrm{O}_{2}$ (between untreated and $\left.\mathrm{H}_{2} \mathrm{O}_{2} ;{ }^{* *} \mathrm{p}<0.005\right) \mathrm{KIKO}$ fibroblasts can be protected significantly by the pre-treatment $(24 \mathrm{~h})$ with $100 \mu \mathrm{M}$ d2-PUFA, $100 \mu \mathrm{M}$ d4-PUFA and the Nrf2inducers (50 nM SFN and $50 \mathrm{nM}$ TBE-31). ${ }^{* *} \mathrm{p}<0.005 ; \mathrm{n}=6$ independent experiments for each condition.

Figure 4: Lipid peroxidation is prevented in YG8R and KIKO fibroblasts by dPUFAs and Nrf2 inducers. A-B. The histogram shows the rate in $\%$ of the ratiometric dye, BODIBY C-11 (581/591), which is a marker for lipid peroxidation. 
The oxidation of the polyunsaturated butadienyl portion of the dye results in a shift of fluorescence. A. YG8R fibroblasts show a significant increase of lipid peroxidation compared to Y47R fibroblasts. The addition of $1 \mathrm{mM} \mathrm{H}_{2} \mathrm{O}_{2}$ further increased lipid peroxidation. Cells pre-treated with d4-PUFA, SFN and TBE-31 significantly recover lipid peroxidation levels towards control values. B. KIKO fibroblasts show an increased lipid peroxidation compared to WT and further increased sensitivity to $\mathrm{H}_{2} \mathrm{O}_{2}$. KIKO fibroblasts also responded well to treatment with d4-PUFA, SFN and TBE-31. ${ }^{*} p<0.05,{ }^{* *} p<0.005,{ }^{* * *} p<0.005 ; n=6$ independent experiments for each condition.

Figure 5: Study of $\Delta \Psi_{\mathrm{m}}$ in YG8R and KIKO fibroblasts. A-B. The histograms show the percentage of basal $\Delta \psi_{m}$ in YG8R (A) and KIKO (B) calculated cell by cell using the Z-stack mode with confocal microscopy. A. YG8R cells show a significant decrease of $\Delta \psi_{\mathrm{m}}$ compared to $\mathrm{Y} 47 \mathrm{R}$ cells, which is recovered by the treatment with d4-PUFA, SFN and TBE-31. B. KIKO show a significant increase of $\Delta \Psi_{\mathrm{m}}$ compared to WT control, but treatment with compounds does not have an effect of this mitochondrial parameter. ${ }^{*} \mathrm{p}<0.05 ; \mathrm{n}=6$ independent experiments. C-D. The graphs show the dynamic response of the $\Delta \Psi_{\mathrm{m}}$ to mitotoxins. C in YG8R cells the potential is decreased compared to control when treated with oligomycin, compared to Y47R; $\mathbf{D}$. while in KIKO cells the potential increases. E-F. The histograms show the summary of values obtained calculation the $\Delta \Psi_{\mathrm{m}}$ in all the genotype with and without protective treatments.

\section{References}


Al-Mahdawi S, Pinto RM, Ruddle P, Carroll C, Webster Z, Pook M (2004) GAA repeat instability in Friedreich ataxia YAC transgenic mice. Genomics 84:301-310.

Al-Mahdawi S, Pinto RM, Varshney D, Lawrence L, Lowrie MB, Hughes S, Webster Z, Blake J, Cooper JM, King R, Pook MA (2006) GAA repeat expansion mutation mouse models of Friedreich ataxia exhibit oxidative stress leading to progressive neuronal and cardiac pathology. Genomics 88:580-590.

Angelova PR, Horrocks MH, Klenerman D, Gandhi S, Abramov AY, Shchepinov MS. (2015) Lipid peroxidation is essential for $\alpha$-synuclein-induced cell death. J Neurochem. Jan 10. doi: 10.1111/jnc. 13024 .

Bulteau, A.-L., O’Neill, H. a, Kennedy, M. C., Ikeda-Saito, M., Isaya, G., \& Szweda, L. I. (2004). Frataxin acts as an iron chaperone protein to modulate mitochondrial aconitase activity. Science (New York, N.Y.), 305(5681), 242-5. doi:10.1126/science.1098991

Barnham, K. J., Masters, C. L., \& Bush, A. I. (2004). Neurodegenerative diseases and oxidative stress. Nature reviews. Drug discovery, 3(3), 205-14. doi:10.1038/nrd1330

Campanella M, Casswell E, Chong S, Farah Z, Wieckowski MR, Abramov AY, Tinker A, Duchen MR. 2008 Regulation of mitochondrial structure and function by the F1Fo-ATPase inhibitor protein, IF1. Cell Metab. Jul;8(1):13-25. doi: 10.1016/j.cmet.2008.06.001. 
Cossée M, Puccio H, Gansmuller A, Koutnikova H, Dierich A, LeMeur M, Fischbeck K, Dollé P, Koenig M. (2000) Inactivation of the Friedreich ataxia mouse gene leads to early embryonic lethality without iron accumulation. Hum Mol Genet. May 1;9(8):1219-26.

Delatycki, M. B., \& Corben, L. a. (2012). Clinical features of Friedreich ataxia. Journal of child neurology, 27(9), 1133-7. doi:10.1177/0883073812448230

Duchen MR, Surin A, Jacobson J. (2003) Imaging mitochondrial function in intact cells. Methods Enzymol. 361:353-89.

Goncalves, S., Paupe, V., Dassa, E. P., \& Rustin, P. (2008). Deferiprone targets aconitase: implication for Friedreich's ataxia treatment. BMC neurology, 8, 20. doi:10.1186/1471-2377$8-20$

González-Cabo, P., Vázquez-Manrique, R. P., García-Gimeno, M. A., Sanz, P., \& Palau, F. (2005). Frataxin interacts functionally with mitochondrial electron transport chain proteins. Human molecular genetics, 14(15), 2091-8. doi:10.1093/hmg/ddi214

Hebert, Michael D. (2008). Targeting the gene in Friedreich ataxia. Biochimie, 90(8), 11319. doi:10.1016/j.biochi.2007.12.005

Herman, D., Jenssen, K., Burnett, R., Soragni, E., Perlman, S. L., \& Gottesfeld, J. M. (2006). Histone deacetylase inhibitors reverse gene silencing in Friedreich's ataxia. Nature chemical biology, 2(10), 551-8. doi:10.1038/nchembio815

Hayes, J. D., McMahon, M., Chowdhry, S. and Dinkova-Kostova, A. T. (2010). Cancer chemoprevention mechanisms mediated through the Keap1-Nrf2 pathway. Antioxid. Redox Signal. 13, 1713-1748. 
Honda, T.; Yoshizawa, H.; Sundararajan, C.; David, E.; Lajoie, M. j.; Favaloro, F. G. Jr.; Janosik, T.; Su, X.; Honda, Y.; Roebuck, B. D.; Gribble, G. W. (2011) Tricyclic compounds containing non-enolizable cyano enones. A novel class of highly potent anti-inflammatory and cytoprotective agents. J. Med. Chem. 54, 1762-1778.

Jones, J., Estirado, A., Redondo, C., Bueno, C., \& Martínez, S. (2012). Human adipose stem cellconditioned medium increases survival of Friedreich's ataxia cells submitted to oxidative stress. Stem cells and development, 21(15), 2817-26. doi:10.1089/scd.2012.0029

Libri V, Yandim C, Athanasopoulos S, Loyse N, Natisvili T, Law PP, Chan PK, Mohammad T, Mauri M, Tam KT, Leiper J,Piper S, Ramesh A, Parkinson MH, Huson L, Giunti P, Festenstein R.(2014) Epigenetic and neurological effects and safety of high-dose nicotinamide in patients with Friedreich's ataxia: an exploratory, open-label, dose-escalation study. Lancet. Aug 9;384(9942):504-13. doi: 10.1016/S0140-6736(14)60382-2. Epub 2014 Apr 30.

Mariotti C, Nachbauer W, Panzeri M, Poewe W, Taroni F, Boesch S. (2013) Erythropoietin in Friedreich ataxia. J Neurochem;126 Suppl 1:80-7. doi: 10.1111/jnc.12301. Review.

Martelli, A., Napierala, M., \& Puccio, H. (2012). Understanding the genetic and molecular pathogenesis of Friedreich's ataxia through animal and cellular models. Disease models \& mechanisms, 5(2), 165-76. doi:10.1242/dmm.008706

Miranda CJ, Santos MM, Ohshima K, Smith J, Li L, Bunting M, Cossée M, Koenig M, Sequeiros J, Kaplan J, Pandolfo M. (2002) Frataxin knockin mouse. FEBS Lett. 13;512(13):291-7. 
Kakhlon, O., Manning, H., Breuer, W., Melamed-Book, N., Lu, C., Cortopassi, G., Munnich, A., et al. (2008). Cell functions impaired by frataxin deficiency are restored by drug-mediated iron relocation. Blood, 112(13), 5219-27. doi:10.1182/blood-2008-06-161919

Koeppen, A. H., \& Mazurkiewicz, J. E. (2013). Friedreich Ataxia : Neuropathology Revised. Journal of neuropathology and experimental neurology, 72(2), 78-90.

Schulz JB, Pandolfo M. (2013) 150 years of Friedreich ataxia: from its discovery to therapy. J Neurochem. Aug;126 Suppl 1:1-3.

Shan, Y., Schoenfeld, R. a, Hayashi, G., Napoli, E., Akiyama, T., lodi Carstens, M., Carstens, E. E., et al. (2013). Frataxin Deficiency Leads to Defects in Expression of Antioxidants and Nrf2 Expression in Dorsal Root Ganglia of the Friedreich's Ataxia YG8R Mouse Model. Antioxidants \& redox signaling, 00(00). doi:10.1089/ars.2012.4537

Shchepinov MS, Chou VP, Pollock E, Langston JW, Cantor CR, Molinari RJ, Manning-Boğ AB. (2011) Isotopic reinforcement of essential polyunsaturated fatty acids diminishes nigrostriatal degeneration in a mouse model of Parkinson's disease. Toxicol Lett.Nov 30;207(2):97-103. doi: 10.1016/j

Soragni E, Miao W, ludicello M, Jacoby D, De Mercanti S, Clerico M, Longo F, Piga A, Ku S, Campau E, Du J, Penalver P, Rai M,Madara JC, Nazor K, O'Connor M, Maximov A, Loring JF, Pandolfo M, Durelli L, Gottesfeld JM, Rusche JR. (2014) Epigenetic therapy for Friedreich ataxia. Ann Neurol.Oct;76(4):489-508. doi: 10.1002/ana.24260. Epub 2014 Sep 16. 
Richardson, T. E., Yu, A. E., Wen, Y., Yang, S.-H., \& Simpkins, J. W. (2012). Estrogen prevents oxidative damage to the mitochondria in Friedreich's ataxia skin fibroblasts. PloS one, 7(4), e34600. doi:10.1371/journal.pone.0034600

Pandolfo, M. (2009). Friedreich ataxia: the clinical picture. Journal of neurology, 256 Suppl , 3-8. doi:10.1007/s00415-009-1002-3

Pandolfo M, Arpa J, Delatycki MB, Le Quan Sang KH, Mariotti C, Munnich A, Sanz-Gallego I, Tai G, Tarnopolsky MA, Taroni F, Spino M, Tricta F. (2014) Deferiprone in Friedreich ataxia: a 6-month randomized controlled trial. Ann Neurol. 76(4):509-21. doi: 10.1002/ana.24248.

Parkinson MH, Boesch S, Nachbauer W, Mariotti C, Giunti P. J Neurochem. (2013) Clinical features of Friedreich's ataxia: classical and atypical phenotypes. J Neurochem. Aug;126 Suppl 1:103-17. doi: 10.1111/jnc. 12317.

Perdomini M, Belbellaa B, Monassier L, Reutenauer L, Messaddeq N, Cartier N, Crystal RG, Aubourg P, Puccio H. (2014) Prevention and reversal of severe mitochondrial cardiomyopathy by gene therapy in a mouse model of Friedreich's ataxia. Nat Med. May;20(5):542-7. doi: 10.1038/nm.3510. Epub 2014 Apr 6.

Perlman, S. L. (2012). A review of Friedreich ataxia clinical trial results. Journal of child neurology, 27(9), 1217-22. doi:10.1177/0883073812453872 
Pook MA, Al-Mahdawi S, Carroll CJ, Cossée M, Puccio H, Lawrence L, Clark P, Lowrie MB, Bradley JL, Cooper JM, Koenig M,Chamberlain S.(2001) Rescue of the Friedreich's ataxia knockout mouse by human YAC transgenesis. Neurogenetics. Oct;3(4):185-93.

Sandi C, Sandi M, Jassal H, Ezzatizadeh V, Anjomani-Virmouni S, Al-Mahdawi S, Pook MA. (2014) Generation and characterisation of Friedreich ataxia YG8R mouse fibroblast and neural stem cell models. PLoSOne. 2014 Feb 21;9(2):e89488. doi: 10.1371/journal.pone.0089488.

Vankan P. (2013) Prevalence gradients of Friedreich's ataxia and R1b haplotype in Europe co-localize, suggesting a common Palaeolithic origin in the Franco-Cantabrian ice age refuge. J Neurochem. Aug;126 Suppl 1:11-20. doi: 10.1111/jnc.12215.

Velasco-Sánchez, D., Aracil, A., Montero, R., Mas, A., Jiménez, L., O’Callaghan, M., Tondo, M., et al. (2011). Combined therapy with idebenone and deferiprone in patients with Friedreich's ataxia. Cerebellum (London, England), 10(1), 1-8. doi:10.1007/s12311-010$0212-7$

Wilson, R. B. (2012). Therapeutic developments in Friedreich ataxia. Journal of child neurology, 27(9), 1212-6. doi:10.1177/0883073812449691

Zamzami N., Marchetti P., Castedo M., Decaudin D., Macho A., Hirsch T., Susin S. A., Petit P. X., Mignotte B. and Kroemer G. (1995) Sequential reduction of mitochondrial transmembrane potential and generation of reactive oxygen species in early programmed cell death. J. Exp. Med. 182, 367-377. 


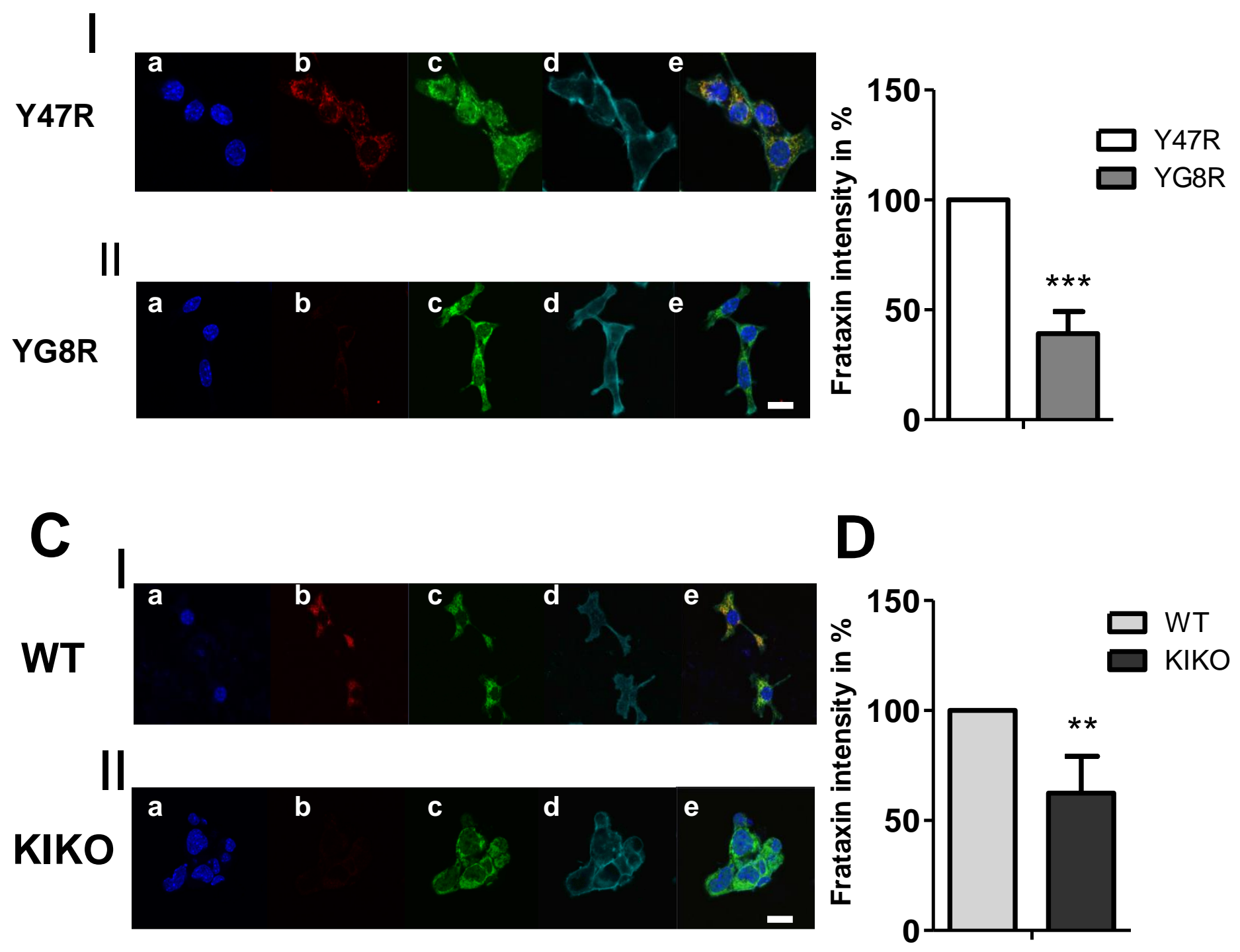

Figure 1 
A

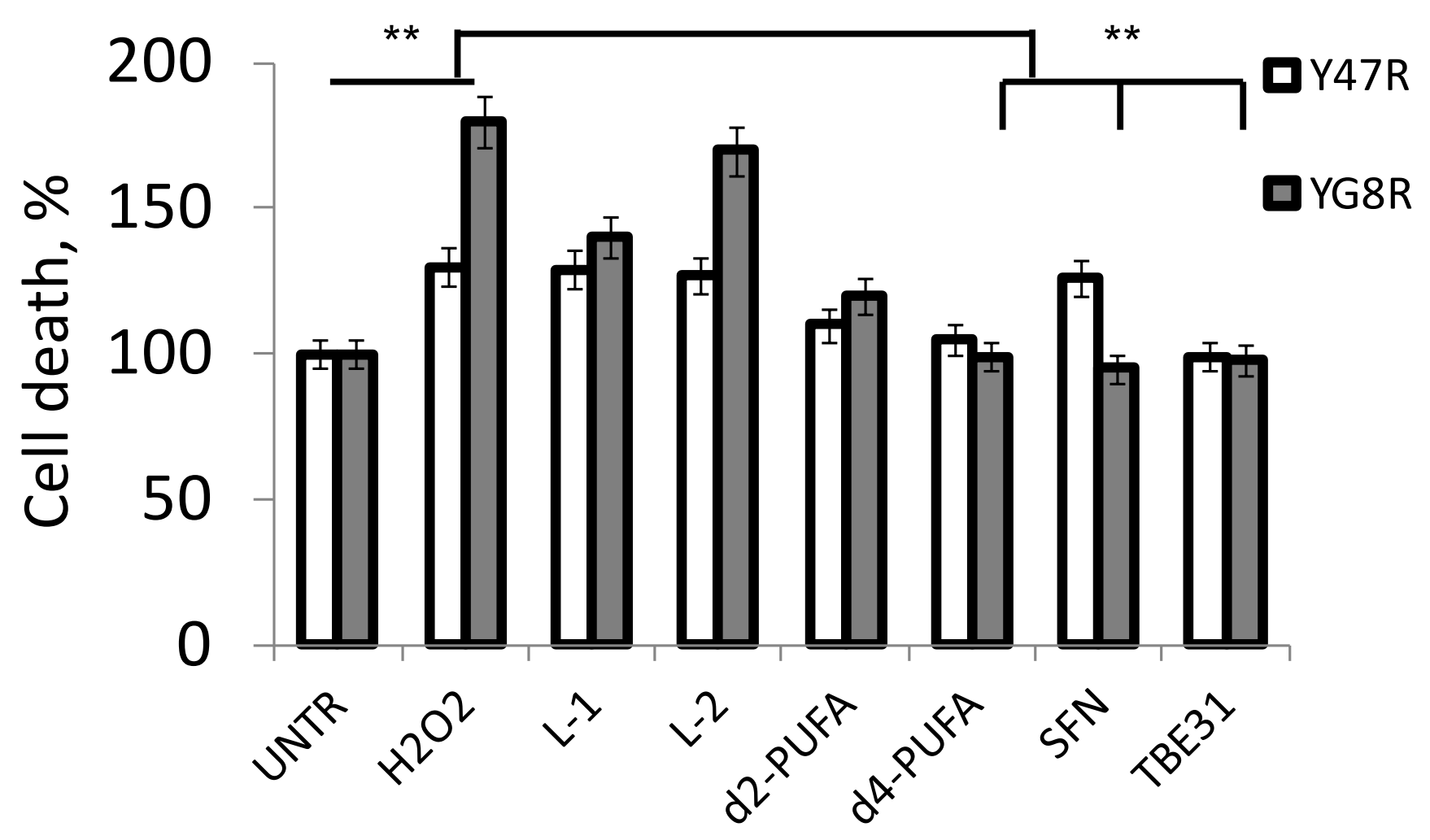

B

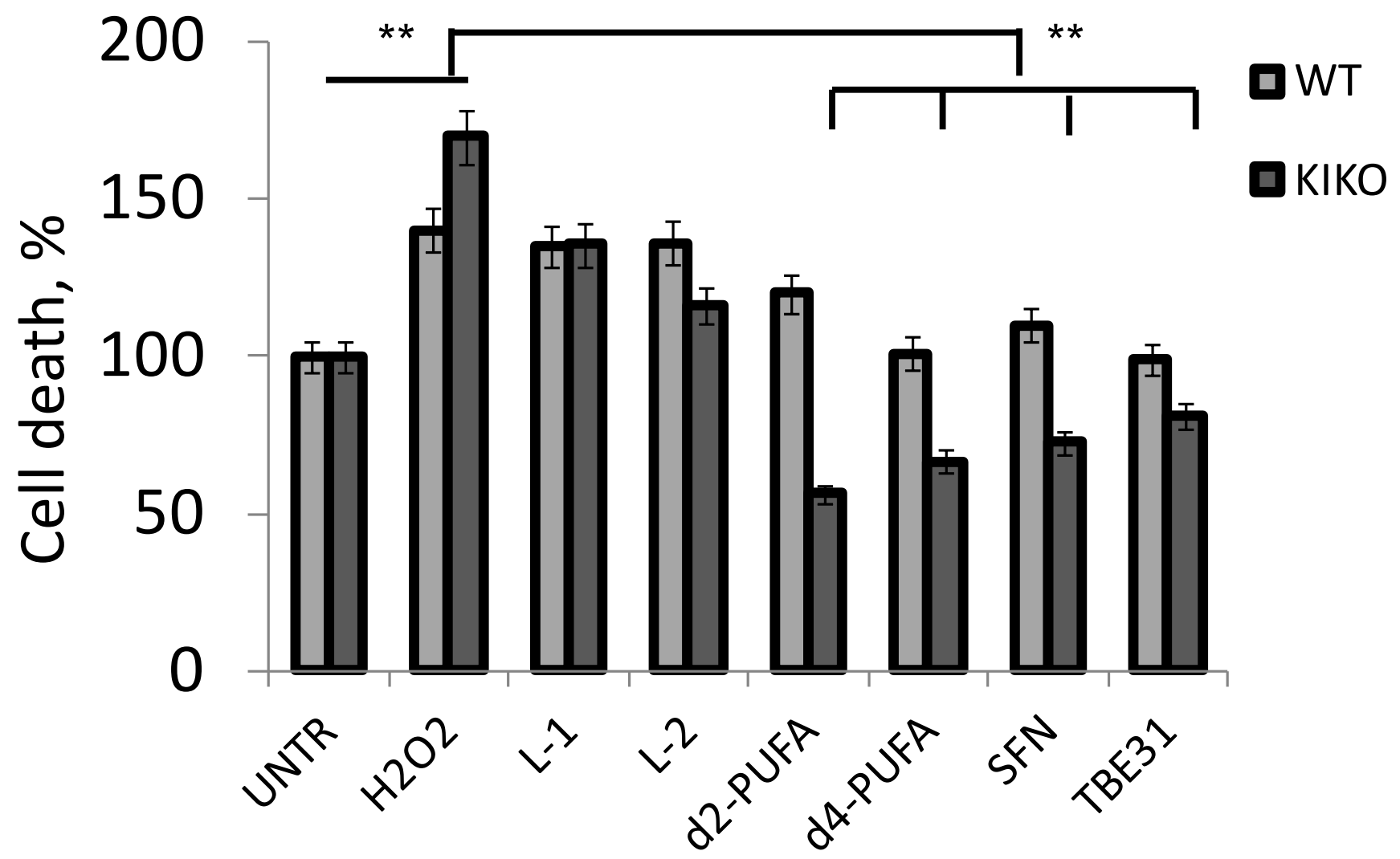

Figure 3 


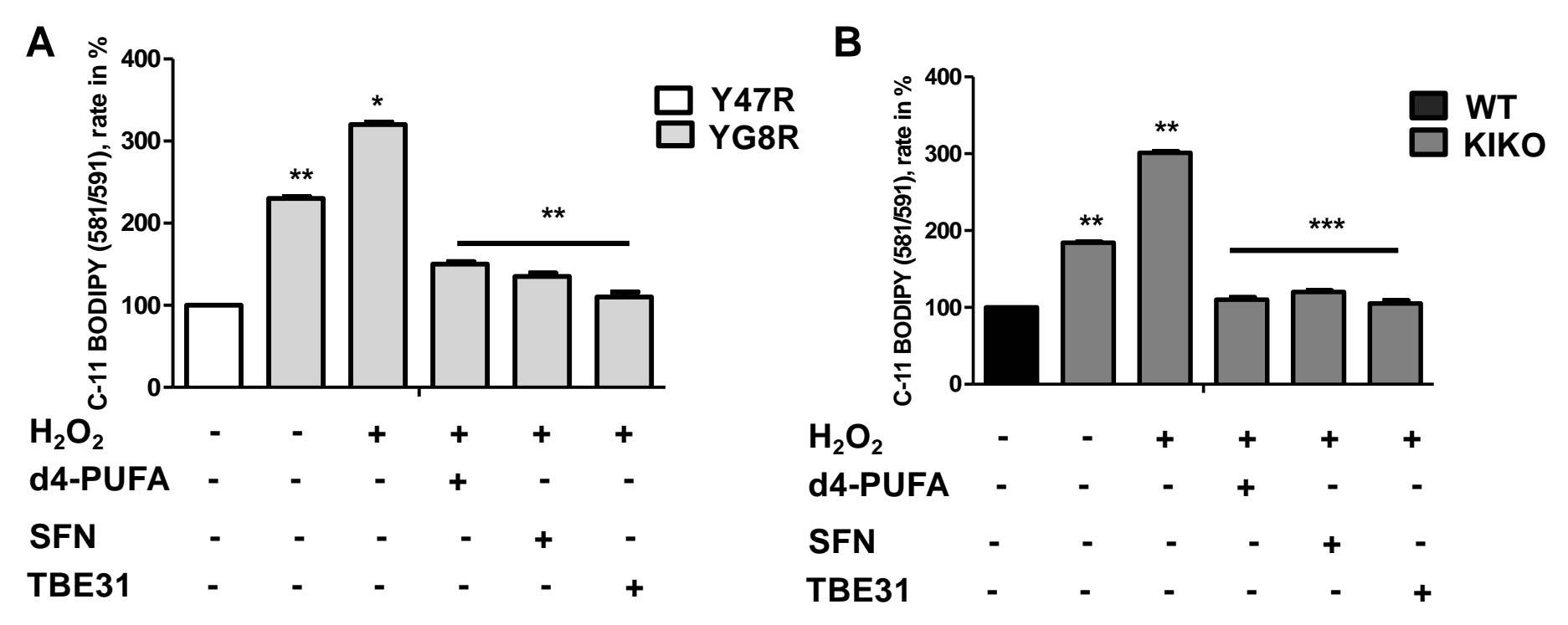

Figure 4 
